\title{
CLÁUSULAS ANTIDISCRIMINATORIAS Y MOTIVOS DE DISCRIMINACIÓN EN LAS CONSTITUCIONES EUROPEAS
}

EDUARDO J. RUIZ VIEYTEZ 


\section{SUMARIO}

1.- Introducción. 2.- La comparación de las cláusulas constitucionales antidiscriminatorias: dificultades metodológicas. 3.- Las cláusulas antidiscriminatorias en el Derecho constitucional comparado europeo. 3.1.- Modelos y estructura. 3.2.- Los motivos de discriminación vigentes. 3.3.- Valoración y nuevos retos. 4.- Conclusión. 5.- Referencias. 


\title{
CLÁUSULAS ANTIDISCRIMINATORIAS Y MOTIVOS DE DISCRIMINACIÓN EN LAS CONSTITUCIONES EUROPEAS ${ }^{1}$
}

\author{
EDUARDO J. RUIZ VIEYTEZ² \\ Universidad de Deusto
}

\section{INTRODUCCIÓN}

Este trabajo tiene por objeto las cláusulas constitucionales europeas en materia de no discriminación, y en particular los factores de discriminación incluidos en las mismas. En particular, queremos analizar y sistematizar la manera en la que los textos constitucionales de Europa abordan el principio no discriminación, estudiando su contenido y detectando patrones comunes. La hipótesis de partida es que los textos actualmente vigentes responden sobre todo a una progresiva acumulación no siempre sistemática de motivos de discriminación que han ido obteniendo legitimidad en el debate social y político, y que aún abordan solo parcialmente discriminaciones en las que se combinan causas diferentes.

Mediante un ejercicio comparativo pretendemos determinar por una parte si existen patrones constitucionales generalizados en la estructura de las cláusulas antidiscriminatorias. Del mismo modo, queremos realizar una comparación y análisis más detallados de los motivos concretos de discriminación que las constituciones mencionan, determinando aquéllos que están presentes con más frecuencia y que pueden considerarse incluidos en el acervo constitucional europeo. Buscamos conocer igualmente el papel que juega la discriminación por razón de sexo dentro de ese conjunto

1 Trabajo realizado en el marco del Proyecto de Investigación del Plan Nacional de I+D+i "La desigualdad compleja en las sociedades plurales. Indicadores para las políticas públicas” (DER201677711-P), subvencionado por el Ministerio de Economía y Competitividad.

2 Profesor del Instituto de Derechos Humanos y Decano de la Facultad de Ciencias Sociales y Humanas de la Universidad de Deusto (Bilbao). Professor at the Human Rights Institute and Dean of the Faculty of Social and Human Sciences at the University of Deusto (Bilbao). Avda. Universidades 24. 48007 Bilbao (Spain). Email: ejruiz@deusto.es 
de criterios, y si ocupa un lugar distinguido en la estructura de las normas citadas. Por último, pretendemos saber si existe una correlación entre motivos de discriminación citados expresamente y diferentes grupos de constituciones, es decir, si puede determinarse una agrupación o evolución en el señalamiento de los motivos de discriminación.

Para ello procederemos a comparar directamente los textos constitucionales. El estudio comparado es básicamente normativo, por lo que la estructura de los textos resulta relevante, así como el momento de su aprobación. Empezaremos señalando los principales problemas metodológicos que implica este intento y realizando una descripción global de las cláusulas estudiadas. En un momento posterior analizaremos con más detalle los motivos de discriminación relacionándolos temáticamente y estudiando su frecuencia. Finalmente valoraremos la eficacia de los tipos básicos de cláusulas a la luz de la realidad actual.

\section{LA COMPARACIÓN DE LAS CLÁUSULAS CONSTITUCIONALES ANTIDISCRIMINATORIAS: DIFICULTADES METODOLÓGICAS}

Si bien puede considerarse que la disciplina académica del Derecho comparado surge a lo largo del siglo XIX (el primer Congreso Internacional de Derecho comparado se celebraría en 1900), la comparación de constituciones no adquirió un impulso importante hasta el último cuarto del siglo $\mathrm{XX}^{3}$. En el ámbito concreto de la comparación de cláusulas constitucionales antidiscriminatorias los trabajos existentes son muy escasos. La primera y más completa recopilación en este ámbito es la realizada por Osin y Porat ${ }^{4}$, que a pesar de su amplitud carece de un análisis comparado del contenido 5 .

La igualdad como derecho aparece en todas las constituciones democráticas modernas pero su interpretación presenta importantes dificultades ${ }^{6}$. En particular, el estudio comparado de las cláusulas antidiscriminatorias vigentes en diferentes textos constitucionales plantea una serie de limitaciones metodológicas que es preciso hacer

${ }^{3}$ GINSBURG, T. y DIXON, R. (eds.)(2011), Comparative Constitutional Law, Cheltenham, Edward Elgar. Pp. 2-3.

${ }^{4}$ OSIN, N. y PORAT, D. (eds.)(2005), Legislating against Discrimination. An international Survey of Anti-Discrimination Norms, Leiden-Boston, Martinus Nijhoff Publishers.

${ }_{5}$ Para el ámbito de la Unión Europea y países vecinos, un estudio muy reciente puede verse en: CHOPIN, I. y GERMAINE, C. (2017), A comparative analysis of non-discrimination law in Europe. The 28 EU Member States, the Former Yugoslav Republic of Macedonia, Iceland, Liechtenstein, Montenegro, Norway, Serbia and Turkey compared (prepared for the European network of legal experts in gender equality and non-discrimination), Brussels, European Commission (Directorate-General for Justice and Consumers).

${ }^{6}$ O’REGAN, K. y FRIEDMAN, N. (2011), "Equality”, en GINSBURG, T. y DIXON, R. (eds.), Comparative Constitutional Law, Cheltenham, Edward Elgar, p. 473. 
constar antes de desarrollar el mismo. Básicamente, nos encontramos con cuatro tipos de dificultades que debemos tener presentes a la hora de realizar este análisis.

En primer lugar, se hace necesario limitar el ámbito de la comparación. Si bien el objeto queda determinado a partir de lo que señala el propio título de este trabajo, el ámbito geográfico (geopolítico) adquiere especial importancia en la medida en que comparamos textos constitucionales y, por tanto, propios de entidades políticas soberanas. Puesto que más allá del peculiar experimento de la Unión Europea no existen propiamente confederaciones o uniones personales o reales de Estados en Europa, se trata de incluir en nuestro análisis exclusivamente Estados independientes y soberanos. Como el ámbito del estudio es el continente europeo, nos vemos obligados a determinar dos cuestiones. Por un lado, cuál es el límite geopolítico de dicho continente. Por otro lado, qué entidades políticas incluimos en el listado a analizar. A la primera pregunta responderemos con un criterio más institucional que de geografía física, considerando que tienen el carácter de europeos, al menos, todos los Estados que actualmente forman parte del Consejo de Europa ( 47 países). Ello incluye, como es sabido a países que geográficamente están fuera de Europa como Georgia, Armenia y Azerbaiyán, así como a países que tienen la mayor parte de su territorio fuera del continente físico, como Rusia, Dinamarca y Turquía.

A la segunda cuestión responderemos en función de que exista un amplio reconocimiento internacional de la entidad política respectiva. Esto es, excluiremos aquellas entidades políticas con vocación estatal que no han obtenido más que un número muy limitado de reconocimientos internacionales, con independencia de que ejerzan o no el control efectivo sobre un territorio y población (Abjasia, Artsaj, Chipre del Norte, Osetia de Sur o Transnistria). Por el contrario, sí incluiremos en nuestro análisis a Kosovo y la Ciudad de Vaticano por tener reconocimiento o relaciones diplomáticas (en el segundo caso como Santa Sede) con más de 100 países miembros de Naciones Unidas, lo que sin duda otorga una apariencia de Estado muy firme. Así pues, al listado de 47 miembros del Consejo de Europa, añadimos los Estados de Kosovo, Vaticano y Bielorrusia para cubrir en la comparación un total de 50 países, de los que 27 serán miembros de la Unión Europea (una vez consumada la salida del Reino Unido de la misma).

La segunda limitación importante de un análisis de textos constitucionales es la relativa a la diversidad lingüística que conlleva. Las constituciones europeas están redactadas en cerca de 40 idiomas diferentes y muy reducidos grupos de ellas son susceptibles de ser comparadas en versión original o en lenguas muy cercanas. Es por ello que se hace preciso utilizar versiones homologadas a través de un solo idioma que necesariamente resulta ajeno al texto original de la mayoría de ellas. El lenguaje que utilizamos para la comparación de los textos es el inglés, y por ello los documentos analizados resultan en su inmensa mayoría traducciones a esta lengua. Esto supone problemas a la hora de identificar los conceptos que las lenguas oficiales incluyen y que no siempre tienen una correspondencia nítida en lengua inglesa o que varían su 
significado en función de contextos socioculturales del lenguaje ${ }^{7}$. En este ejercicio de traducción se pueden producir, lógicamente, inexactitudes, aunque ello no debe afectar severamente al ejercicio de estudiar los grupos de motivos de discriminación que más preocupan a los constituyentes europeos.

Como tercera limitación, es necesario notar que no solamente son diferentes las lenguas en las que las distintas constituciones son redactadas, sino también las características principales de las constituciones que se comparan. Es verdad que la mayoría de ellas responden a tradiciones jurídico-políticas muy similares y que un número muy amplio de las mismas han sido aprobadas durante las últimas décadas. La cultura política y jurídica que está en la base de ellas no resulta tan diferente, pero se trata de textos diferentes, con sus respetivas estructuras y condiciones de redacción. Es innegable que ello afecta también a la comparación en la medida en que los estilos, las intenciones o las posibilidades de cada texto varían. Desde el punto de vista de la estructura, las diferencias más notables las ofrecen los casos en los que no tenemos una constitución formal escrita en sentido estricto o nos encontramos con diferentes textos de rango constitucional que deben ser tenidos en cuenta ${ }^{8}$. Así, si bien en el caso del Reino Unido se hace particularmente difícil incluirlo en el análisis, para la República Checa atendemos a lo que establece la Carta de Derechos y Libertades Fundamentales y para el caso de Suecia al Instrumento de Gobierno.

Finalmente, es preciso también recordar que los 50 Estados sobre los que aplicamos nuestra comparación comparten su pertenencia a Europa pero ofrecen realidades políticas muy diferenciadas. Por un lado, porque la lista de países incluye microestados de tamaño muy reducido (Vaticano, San Marino, Andorra, Liechtenstein, Mónaco, Malta, Luxemburgo o Montenegro) frente al país más extenso del planeta (Rusia). Por otra parte, porque si bien la mayor parte de los países responden a sistemas constitucionales de democracia representativa, algunos de ellos no pueden considerarse democracias ni siquiera en un sentido amplio (lo que impide su pertenencia al Consejo de Europa) como Vaticano o Bielorrusia. Esto no obstante, es igualmente cierto que la mayor parte de los Estados en comparación ofrecen muchas más similitudes que diferencias desde el punto de vista jurídico constitucional, por lo que la muestra es tan amplia como válida para obtener conclusiones de la misma.

7 PEGORANO, L. y RINELla, A. (2007), Diritto Pubblico Comparato. Profili metodologici, Padova, CEDAM., pp. 102-103; OSIN, N. y PORAT, D., cit., p. xix.

8 OSIN, N. y PORAT, D., cit., p. xix. 


\section{LAS CLÁUSULAS ANTIDISCRIMINATORIAS EN EL DERECHO CONSTITUCIONAL COMPARADO EUROPEO}

\subsection{Modelos y estructura}

La cláusula tipo en materia de antidiscriminación tiene una estructura muy sencilla. En una primera frase proclama el derecho a la igualdad o a la igualdad ante la ley de todas las personas (o de los ciudadanos o nacionales) y en un segundo enunciado excluye toda posible discriminación sobre la base de un determinado listado de motivos. Sobre este esquema básico algunas constituciones introducen variaciones de diverso tipo o contenido.

La presencia de una cláusula de este tipo en las constituciones europeas es muy generalizada. Solamente podríamos excluir países muy concretos. En el caso del Reino Unido, la peculiar naturaleza de su constitución hace que no podamos aludir a una disposición concreta ${ }^{9}$. Tampoco encontramos una cláusula comparable en el Acta Constitucional de Dinamarca ni en la Ley Fundamental del Estado de la Ciudad del Vaticano. La Constitución de Luxemburgo, por su parte, proclama en su artículo 10 que los luxemburgueses con iguales ante la ley, pero no incluye una disposición específicamente antidiscriminatoria, algo que sucede también en la Constitución de Mónaco. Por el contrario, la Constitución de Irlanda incluye la igualdad ante la ley sin hacer referencia a la no discriminación, pero ésta sí es prevista cuando se habla específicamente de la libertad de conciencia y religión (artículo 44). De la misma manera, la Constitución de Grecia incorpora un listado de motivos que excluyen la discriminación en referencia al derecho a la vida, honor y libertad (artículo 5.2), y la Constitución de Montenegro lo hace al limitar las posibles restricciones a derechos derivadas de la aplicación de estados de emergencia (artículo 25).

Así, son 42 los Estados analizados que incluyen una cláusula antidiscriminatoria específica en sus constituciones. Pero no en todos ellos se incluye un listado de motivos de discriminación que podamos comparar. En efecto, hay 6 constituciones que, teniendo una cláusula antidiscriminatoria, no incorporan un listado de motivos de discriminación: Bélgica, Letonia, Polonia (dentro de la UE), Bielorrusia, Liechtenstein y Noruega (fuera de la UE). De este modo, 36 constituciones incluyen tanto una cláusula tipo como un listado de motivos de discriminación. Si a efectos de comparar los listados sumamos los que incorporan las constituciones de Irlanda, Grecia y Mon-

9 En el caso de Inglaterra y Gales, el principio antidiscriminatorio se incorporó al ordenamiento jurídico en 1965 con la aprobación de la Race Relations Act. Progresivamente, en 1970 se aprobaría la Equal Pay Act, en 1975 la Sexual Discrimination Act, en 1995 la Disability Discrimination Act y en 2004 la Gender Recognition Act. Todas estas normas dieron paso en 2006 a una única Equality Act, que fue reformada en 2010. Según el primer capítulo de esta ley estatutaria, las "características protegidas" o motivos sobre los que ese aplica expresamente el principio de no discriminación son la edad, la discapacidad, la asignación de género, el matrimonio o pareja civil, la raza, la religión o creencias, el sexo y la orientación sexual.

(C) UNED. Revista de Derecho Politico 
tenegro a las que ya hemos aludido, nos encontramos con un total de 39 listados de motivos de discriminación para el ejercicio comparativo.

En este punto, algunos autores distinguen tres grupos de países, en función de si establecen o no listados de motivos de discriminación o factores de protección:

a) Ordenamientos que incluyen una lista exhaustiva y cerrada de motivos.

b) Ordenamientos que recogen el principio o derecho a la igualdad, pero sin establecer un listado de motivos que pueden dar lugar a discriminación.

c) Ordenamientos con un listado que no es exhaustivo o cerrado. Esto se concreta básicamente a través de dos técnicas diferentes:

a. Incluyendo un motivo residual genérico que incorpore cualquier otra posible condición o factor social, personal, etc.

b. mediante una modulación al comienzo del listado que aclare que es ejemplificativo, con expresiones como "en particular...", "incluyendo tales como...", etc.

Por supuesto, la inexistencia de un listado constitucional no cierra la puerta a que el mismo pueda estar residenciado en otra fuente jurídica, como sería el caso del Reino Unido que, sin tener una cláusula constitucional comparable, pertenecería al primero de los grupos citados. No obstante, se trata en Europa más de una excepción que de una regla generalizada.

La principal diferencia entre los modelos citados es el margen de intervención del que disponen los jueces a la hora de aplicar las normas antidiscriminatorias. En los supuestos de listados cerrados su actuación se limitará a considerar exclusivamente los motivos incluidos, mientras que los otros dos modelos dejan a los jueces un margen mucho más amplio de intervención en la determinación o creación de motivos nuevos ${ }^{10}$.

En nuestra comparación, los Estados sin listado en su Constitución constituyen una minoría de 11 respecto a 50. Dentro de las 39 constituciones que sí incluyen enumeraciones, la inclusión de un motivo residual abierto o de una expresión que deja abierto el listado es una técnica seguida por 23 países, mientras que los restantes 16 textos constitucionales pertenecerían a la categoría de cláusulas con listados cerrados. La distribución de cada Constitución conforme a estos modelos puede apreciarse en la siguiente tabla:

10 FREDMAN, S. (2011), Discrimination Law, 2nd ed., Oxford, Oxford University Press, p. 113; SOLANKE, I., cit., pp. 43-49. Ambos autores coinciden en la categorización de los países que analizan, con una significativa excepción: Estados Unidos, país que no hace parte de nuestro estudio. 
Tabla 1: Constituciones europeas según la estructura del listado de motivos de discriminación

\begin{tabular}{|c|c|c|c|}
\hline \multicolumn{2}{|l|}{ Constituciones } & UE & No UE \\
\hline Sin listado & 11 & $\begin{array}{l}\text { Bélgica, Dinamarca, Letonia, } \\
\text { Luxemburgo, Polonia }\end{array}$ & $\begin{array}{c}\text { Bielorrusia, Liechtenstein, } \\
\text { Mónaco, Noruega, Reino } \\
\text { Unido, Vaticano, }\end{array}$ \\
\hline Con listado cerrado & 16 & $\begin{array}{c}\text { Alemania, Austria, Bulgaria, } \\
\text { Francia, Grecia, Irlanda, Italia, } \\
\text { Lituania, Malta, Portugal, } \\
\text { Rumanía }\end{array}$ & $\begin{array}{c}\text { Azerbaiyán, Georgia, } \\
\text { Macedonia, Moldavia, San } \\
\text { Marino, }\end{array}$ \\
\hline $\begin{array}{l}\text { Con listado incluyendo } \\
\text { motivo residual }\end{array}$ & 20 & $\begin{array}{l}\text { Chequia, Chipre, Croacia, } \\
\text { Eslovaquia, Eslovenia, España, } \\
\text { Estonia, Finlandia, Hungría, } \\
\text { Países Bajos, Suecia, }\end{array}$ & $\begin{array}{c}\text { Andorra, Armenia, Bosnia, } \\
\text { Islandia, Kosovo, Montenegro, } \\
\text { Rusia, Ucrania, Turquía }\end{array}$ \\
\hline $\begin{array}{l}\text { Con listado } \\
\text { ejemplificativo }\end{array}$ & 3 & & Albania, Serbia, Suiza \\
\hline Total & 50 & 27 & 23 \\
\hline
\end{tabular}

Por lo que respecta a la titularidad del derecho a la igualdad o a la no discriminación, una amplia mayoría de las cláusulas analizadas se refieren a todas las personas, mientras que en otros 16 casos la formulación del sujeto es más restrictiva. En estos casos, la mayor parte de las veces la expresión utilizada es la de "ciudadanos" lo que al menos en un principio parece restringir la titularidad a los nacionales del país en cuestión, aunque el concepto de ciudadano puede encerrar diferentes significados. Así, "ciudadanos" es el término utilizado en las constituciones de Francia, Irlanda, Italia, Rumanía, Bulgaria (dentro de la UE), Moldavia, Macedonia y Liechtenstein (fuera de la UE), mientras que en el caso austríaco el término usado es el de "nacionales". En cambio, las constituciones de España, Bélgica, Luxemburgo, Grecia y Mónaco utilizan el respetivo gentilicio nacional para referirse al titular del derecho a la igualdad.

Otro aspecto relevante en cuanto a la estructura de las cláusulas de no discriminación es la presencia de la igualdad entre géneros, más allá de que el propio género o sexo constituya uno de los motivos de discriminación expresamente aludidos. En nuestra comparación hemos podido comprobar que hasta 24 constituciones contienen referencias explícitas a la igualdad entre géneros o la no discriminación entre los mismos. Ahora bien, estas cláusulas a su vez pueden revestir formatos diferentes o abordar ámbitos distintos. Por un lado, existen cláusulas generales que aluden expresamente a la igualdad o no discriminación entre géneros para cualquier ámbito en un total de 19 constituciones (Alemania, Austria, Bélgica, Finlandia, Grecia, Luxembur- 
go, Malta, Polonia y Suecia en la UE; Armenia, Azerbaiyán, Islandia, Liechtenstein, Montenegro, Rusia, Serbia, Suiza, Turquía y Ucrania fuera de la UE). Al mismo tiempo, hay otras 11 constituciones que incluyen referencias específicas a la igualdad o no discriminación entre géneros para algún ámbito o derecho concreto. Una lectura de estas cláusulas más específicas nos permite comprobar que aluden fundamentalmente a tres ejes que podríamos denominar con los adjetivos institucional, laboral y educativo.

Un primer grupo de constituciones incorpora previsiones de igualdad entre sexos en la presencia institucional, mandatos o funciones públicas. Así, en el caso de la constitución francesa, su artículo primero obliga a que la ley favorezca el acceso igual de mujeres y hombres a los mandatos y funciones electivas, así como a las responsabilidades profesionales y sociales. En Italia, el artículo 51 señala que los ciudadanos de ambos sexos son elegibles para los oficios y posiciones públicas en igualdad, obligando a la República a adoptar medidas específicas que promuevan oportunidades iguales entre hombres y mujeres. También la Constitución de Rumanía incorpora en su artículo 16 la previsión de que el Estado garantizará la igualdad de oportunidades entre hombres y mujeres a la hora de ocupar posiciones públicas, al igual que hace la Constitución de Polonia, en su artículo 33.2. La Constitución de Kosovo se refiere en su artículo 71 a la Asamblea parlamentaria, cuya composición deberá respetar los "principios de igualdad de género reconocidos internacionalmente". Finalmente, la Constitución de Bélgica señala en el artículo 11bis que hombres y mujeres pueden ejercer sus derechos en igualdad, y en particular promueve su acceso igual a los mandatos electivos y públicos, recordando que los diferentes gobiernos del país deben incluir personas de ambos sexos.

En un segundo ámbito pueden agruparse disposiciones relativas a la igualdad entre sexos que se refieren al plano laboral. La Constitución de Italia recoge en su artículo 37 que las mujeres trabajadoras tienen derecho a la misma retribución que los hombres por trabajos equivalentes, y que sus condiciones de trabajo deben permitir a las mujeres cumplir su papel esencial en la familia y asegurar una adecuada protección para las madres y los hijos. Desde luego, resulta discutible que una cláusula que asigna específicamente a las mujeres un rol especial en el ámbito familiar o filial pueda considerarse como una cláusula de igualdad y no discriminación entre géneros, pero en este análisis mencionaremos las cláusulas presentes, más allá de su idoneidad o eficacia. Esta idea de unir el género femenino con el ámbito familiar se encuentra también en el artículo 8 de la Constitución de Suiza, si bien de manera menos expresa. El mismo precepto exige igualdad en el lugar de trabajo y misma remuneración para hombres y mujeres por trabajos de igual valor. La del derecho a una remuneración igual entre hombres y mujeres por el mismo o equivalente trabajo es la cláusula más mencionada en este ámbito laboral. La incluyen expresamente, además de las dos citadas, las constituciones de Bielorrusia (artículo 42.2), Finlandia (artículo 6), Malta (artículo 14), Polonia (artículo 33) y Ucrania (artículo 24). En añadidura, la Constitución de Polonia menciona dentro de la igualdad entre hombres 
y mujeres el acceso al empleo y la promoción laboral (artículo 33). También Bielorrusia incluye la promoción laboral (artículo 32.5) y la Constitución de Ucrania se extiende en mayor medida en este ámbito aludiendo a la previsión de derechos y condiciones específicas por razón de maternidad (artículo 24.3).

Por último, algunas de estas mismas constituciones incluyen referencias expresas a la igualdad o no discriminación entre géneros en el plano educativo o cultural. Junto a una mención muy breve en el caso de la Constitución de Suiza (artículo 8), la Constitución de Polonia establece igualdad de derechos entre hombres y mujeres para los ámbitos "familiar, político, social y económico" (artículo 33.1), insistiendo más adelante en particular en la educación (artículo 33.2). Fuera de la UE, la Constitución de Bielorrusia señala que las mujeres tendrán igualdad de derechos en lo concerniente a la educación y formación profesional, así como en las esferas cultural y sociopolítica (artículo 32.5) y la Constitución de Ucrania incluye la igualdad de mujeres y hombres en la actividad cultural y en la obtención de educación y formación profesional.

En total, son 24 las constituciones de nuestro estudio que incorporan referencias expresas a la igualdad o no discriminación entre los dos géneros más allá de lo establecido en los listados respectivos de motivos de discriminación. De ellas, 12 corresponden a países miembros de la UE y otras 12 a países no miembros. 19 constituciones incorporan una cláusula genérica para todos los ámbitos al mismo tiempo y 11 constituciones incluyen referencias a ámbitos concretos, mientras que 6 constituciones contienen tanto una referencia genérica como alguna cláusula ligada a un ámbito específico.

Tabla 2: Constituciones europeas que incluyen referencias expresas genéricas o específicas a la igualdad entre hombres y mujeres

\begin{tabular}{|c|c|c|c|}
\hline \multicolumn{2}{|c|}{$\begin{array}{l}\text { Constituciones con } \\
\text { referencias explícitas a la } \\
\text { igualdad entre sexos }\end{array}$} & UE & No UE \\
\hline Referencia genérica & 13 & $\begin{array}{c}\text { Alemania, Austria, } \\
\text { Luxemburgo, Grecia, Suecia }\end{array}$ & $\begin{array}{c}\text { Armenia, Azerbaiyán, Islandia, } \\
\text { Liechtenstein, Montenegro, } \\
\text { Rusia, Serbia, Turquía }\end{array}$ \\
\hline $\begin{array}{l}\text { Referencia } \\
\text { específica }\end{array}$ & 5 & Francia, Italia, Rumanía & Bielorrusia, Kosovo \\
\hline $\begin{array}{c}\text { Referencia genérica } \\
\text { y específica }\end{array}$ & 6 & $\begin{array}{l}\text { Bélgica, Finlandia, Malta, } \\
\text { Polonia }\end{array}$ & Suiza, Ucrania \\
\hline Total & 24 & 12 & 12 \\
\hline
\end{tabular}




\subsection{Los motivos de discriminación vigentes}

Como hemos señalado en el apartado anterior, un total de 22 constituciones de países miembros de la UE y 17 de países europeos no miembros de la UE contienen un listado de motivos de discriminación. Sobre la base de la comparación de estos 39 listados pretendemos identificar cuáles son los motivos de discriminación más habitualmente incorporados a las cláusulas antidiscriminatorias y detectar qué factores se consideran en nuestra cultura constitucional más proclives a conducir a una discriminación. Sabemos que un número significativo de constituciones (20) incluye un motivo final de tipo residual en el que se alude a "cualquier otra circunstancia o condición", "otras características" u "otros estatus". Esta cláusula residual no invalida el análisis del resto de los motivos puesto que éstos siguen dándonos la lista de los que las constituciones reconocen o consideran más relevante y necesario mencionar a fin de evitar la discriminación.

Las dificultades resultantes de trabajar con textos traducidos y la variedad de culturas constitucionales de los países europeos, conducen a un primer listado de motivos extraordinariamente extenso. Incluso agrupando motivos que sin tener el mismo tenor literal contienen las mismas o muy similares palabras, el número de posibles motivos de discriminación se encuentra en torno a 50 factores diferentes en el conjunto de las 39 constituciones aludidas. Este primer listado incluye los siguientes motivos referidos según el número de constituciones en las que son mencionados:

Tabla 3: Motivos de discriminación referidos expresamente en las constituciones europeas según el número de constituciones que los incluyen

\begin{tabular}{|l|l|}
\hline 1. sex/gender & 36 \\
\hline 2. race & 34 \\
\hline 3. religion/religious affiliation/ religious condition & 31 \\
\hline 4. language/ linguistic affiliation & 28 \\
\hline $\begin{array}{l}\text { 5. political opinion/ political condition/ political conviction/ political belief/ political } \\
\text { views }\end{array}$ & 26 \\
\hline 6. any other ground/ other characteristics/ other status & 20 \\
\hline 7. colour/ colour of skin & 16 \\
\hline 8. property/ property status & 16 \\
\hline 9. opinion/ other opinion/other views & 15 \\
\hline 10. birth & 14 \\
\hline 11. national origin & 14 \\
\hline
\end{tabular}




\begin{tabular}{|c|c|}
\hline 12. origin/ place of origin & 13 \\
\hline 13. social origin & 13 \\
\hline 14. ethnicity/ ethnic identity/ ethnic origin/ethnic affiliation & 10 \\
\hline 15. nationality & 10 \\
\hline 16. religious opinion/ religious conviction/ religious belief & 9 \\
\hline 17. social status/ social position & 9 \\
\hline 18. disability/ functional disability/ physical, mental or psychological disability & 9 \\
\hline 19. conviction/other conviction & 8 \\
\hline 20. ideological belief/ philosophical belief/ belief/ ideological convictions/ other belief & 8 \\
\hline 21. social condition/ social circumstance/ social affiliation/ social belonging & 7 \\
\hline 22. economic situation/ economic condition/ financial position & 7 \\
\hline 23. political affiliation/membership in political parties & 5 \\
\hline 24. education & 5 \\
\hline 25. age & 5 \\
\hline 26. sexual orientation & 4 \\
\hline 27. creed/ faith & 4 \\
\hline 28. belonging to/ association with/ affiliation to a national minority & 4 \\
\hline 29. personal condition/ personal circumstance/ personal status & 4 \\
\hline 30. place of residence & 3 \\
\hline 31. membership of (other) public associations/organizations & 2 \\
\hline 32. official status & 2 \\
\hline 33. parentage & 2 \\
\hline 34. relation to any community & 2 \\
\hline 35. class & 2 \\
\hline 36. genetic features & 1 \\
\hline 37. gender identity & 1 \\
\hline 38. worldview & 1 \\
\hline 39. membership in trade unions & 1 \\
\hline 40. sect & 1 \\
\hline 41. culture & 1 \\
\hline 42. estate & 1 \\
\hline 43. ancestry & 1 \\
\hline
\end{tabular}

(C) UNED. Revista de Derecho Politico 


\begin{tabular}{|l|l|}
\hline 44. homeland & 1 \\
\hline 45. national affiliation & 1 \\
\hline 46. belonging to a minority group & 1 \\
\hline 47. occupation & 1 \\
\hline 48. material standing & 1 \\
\hline 49. way of life & 1 \\
\hline 50. health & 1 \\
\hline
\end{tabular}

Pero el análisis principal aún necesita de otro ejercicio, toda vez que esta mínima agrupación "léxica" de los motivos de discriminación deja un panorama muy abierto y con motivos muy cercanos entre sí desde la perspectiva semántica. Esto nos lleva a agrupar varios de los motivos mencionados en categorías más amplias por su proximidad semántica. Así, por ejemplo, haremos con los términos que aluden a aspectos fenotípicos visibles de las personas como "raza", "color de piel", "color" o "rasgos genéticos". Lo mismo haremos con otros motivos que aluden a los mismos factores como las diferentes formas de aludir a la religión, creencias religiosas, convicción religiosa, pertenencia religiosa, fe, culto o términos similares. Otro de los grupos girará en torno a las convicciones, opiniones o pertenencias de base expresamente política, dejando en otra agrupación a las creencias no expresamente religiosas, ideologías, opiniones o adscripciones filosóficas, aun sabiendo que la frontera semántica entre este último grupo y los dos anteriores puede resultar en algunos casos difusa.

Pueden también relacionarse en torno a varios supermotivos las alusiones al origen nacional (origin/place of origin/homeland/national origin); a la condición social (social condition/social circumstance/social affiliation/social belonging/social origin/ social status/social position/class), a la condición económica (economic situation/ economic condition/financial position/material standing/property/property status ), al elemento étnico (ethnicity/ethnic identity/ethnic origin/ethnic affiliation) y a la salud (health/disability/functional disability/physical, mental or psychological disability). Finalmente, se pueden agrupar igualmente diversas alusiones a la pertenencia a minorías (belonging to/association with/affiliation to a national minority/minority group/community), el estatus o condición personal (personal condition/personal circumstance/personal status/official status/estate), o la pertenencia a entidades políticas o sindicales (membership of — other - public associations/organizations/trade union/ political parties/political affiliation). Podemos del mismo modo equiparar la "ocupación" al "modo de vida, así como la "nacionalidad" con la "filiación nacional".

Con este ejercicio de agrupación por similitud semántica, habríamos conseguido rebajar sensiblemente el listado anterior a la mitad, pasando de 50 categorías diferentes a solo 25 , con la frecuencia que se señala en la siguiente tabla: 
Tabla 4: Agrupaciones de los motivos de discriminación referidos expresamente en las constituciones europeas según el número de constituciones en las que aparecen

\begin{tabular}{|c|c|c|c|c|}
\hline \multirow{2}{*}{$\begin{array}{l}\text { Agrupaciones } \\
\text { de conceptos }\end{array}$} & \multirow[t]{2}{*}{ Conceptos citados en las Constituciones } & \multicolumn{3}{|c|}{ Constituciones } \\
\hline & & $\mathrm{UE}$ & No & Total \\
\hline Religión & $\begin{array}{l}\text { religion/religious affiliation/religious condition/creed/ } \\
\text { faith/sect/religious opinion/religious conviction/religious } \\
\text { belief }\end{array}$ & 21 & 18 & 39 \\
\hline Sexo & sex / gender/gender identity & 19 & 17 & 36 \\
\hline Fenotipo & race/skin colour/colour/genetic features & 19 & 16 & 35 \\
\hline Convicción & $\begin{array}{l}\text { opinion/other opinion/other views/worldview/ conviction/ } \\
\text { other conviction/ideological belief/philosophical belief/ } \\
\text { belief/ideological convictions/other belief }\end{array}$ & 13 & 15 & 28 \\
\hline Lengua & language/linguistic affiliation & 15 & 13 & 28 \\
\hline Origen & origin / place of origin/homeland/national origin & 15 & 12 & 28 \\
\hline Opinión política & $\begin{array}{l}\text { political opinion/political condition/political conviction/ } \\
\text { political belief/political views }\end{array}$ & 13 & 13 & 26 \\
\hline Condición social & $\begin{array}{l}\text { social condition/ social circumstance/ social affiliation/ } \\
\text { social belonging/social origin/ social status/social position/ } \\
\text { class }\end{array}$ & 14 & 12 & 26 \\
\hline $\begin{array}{l}\text { Condición } \\
\text { económica }\end{array}$ & $\begin{array}{l}\text { economic situation/ economic condition/ financial } \\
\text { position/material standing/ property/property status }\end{array}$ & 9 & 14 & 23 \\
\hline Otro & any other ground/other characteristics/other status & 11 & 9 & 20 \\
\hline Nacimiento & birth & 8 & 6 & 14 \\
\hline Nacionalidad & nationality/national affiliation & 6 & 5 & 11 \\
\hline Etnia & ethnicity/ ethnic identity/ethnic origin/ ethnic affiliation & 5 & 5 & 10 \\
\hline Salud & $\begin{array}{l}\text { health/disability/functional disability/ physical, mental or } \\
\text { psychological disability }\end{array}$ & 5 & 4 & 9 \\
\hline Minoría & $\begin{array}{l}\text { belonging to/association with/ affiliation to a national } \\
\text { minority/ minority group/community }\end{array}$ & 3 & 4 & 7 \\
\hline Estatus & $\begin{array}{l}\text { personal condition/personal circumstance/ personal status/ } \\
\text { official status/estate }\end{array}$ & 5 & 2 & 7 \\
\hline Membrecía & $\begin{array}{l}\text { membership of (other) public associations/ organizations/ } \\
\text { trade union/political parties/ political affiliation }\end{array}$ & 3 & 3 & 6 \\
\hline Educación & Education & 4 & 1 & 5 \\
\hline Edad & age & 2 & 3 & 5 \\
\hline
\end{tabular}

(C) UNED. Revista de Derecho Político 
EDUARDO J. RUIZ VIEYTEZ

\begin{tabular}{|c|c|c|c|c|}
\hline \multirow{2}{*}{$\begin{array}{l}\text { Agrupaciones } \\
\text { de conceptos }\end{array}$} & \multirow[t]{2}{*}{ Conceptos citados en las Constituciones } & \multicolumn{3}{|c|}{ Constituciones } \\
\hline & & UE & No & Total \\
\hline $\begin{array}{l}\text { Orientación } \\
\text { sexual }\end{array}$ & sexual orientation & 3 & 1 & 4 \\
\hline Residencia & place of residence & 0 & 3 & 3 \\
\hline Parentesco & Parentage & 1 & 1 & 2 \\
\hline Ocupación & occupation/way of life & 0 & 2 & 2 \\
\hline Cultura & culture & 0 & 1 & 1 \\
\hline Ascendencia & Ancestry & 1 & 0 & 0 \\
\hline
\end{tabular}

A partir de este punto, realizar nuevas agrupaciones de conceptos por cercanía semántica no nos aporta mucha más claridad en el análisis. Por un lado, porque los conceptos que pudieran agruparse se repiten dentro de las mismas constituciones, de forma que los números finales no cambian sustancialmente. Por otro lado, porque en la agrupación corrernos el riesgo de olvidar o diluir la potencialidad de alguno de los motivos de discriminación. Así, por ejemplo, la orientación sexual es citada expresamente solo por 4 constituciones, en lo que constituye un reconocimiento de la relativamente reciente tendencia de considerar que las opciones sexuales pueden constituir motivo de discriminación. Pero sería un error agrupar este motivo con el más amplio que alude al género, sexo o identidad de género. Mientras que aquél busca claramente prohibir las discriminaciones basadas en opciones no exclusivamente heterosexuales, éste se refiere a las diferenciaciones entre identidades de género (hombre, mujer y otras identidades establecidas sobre el mismo eje).

Más cuestionable es una posible agrupación, o una clasificación diferente, de los motivos citados en torno a las opiniones, creencias o convicciones en general, incluyendo las ideológicas o filosóficas y las convicciones u opiniones con adjetivo expresamente político. Ambos grupos ocupan los lugares cuatro y séptimo de nuestra tabla, pero en el caso de fusionarlos en un único megagrupo, no superarían la posición cuarta, si bien aumentaría el número de presencia total en constituciones, desde 28 y 26 a un total de 35 (de las que 18 son de la UE y 17 extracomunitarias). Finalmente, si entendemos el motivo de origen en clave no solamente geográfica sino ligada a un territorio o comunidad política o cultural concreta, podríamos agrupar este motivo (sexto en nuestra clasificación) con los referidos a la nacionalidad o la pertenencia étnica, e incluso con la cultura. Se trataría así de un origen mucho más amplio que lo que la propia palabra indica, si bien es posible que con la misma en muchas ocasiones se aluda realmente a lo que dicho origen étnico o nacional supone de diferenciación (en sentido minoritario) de una determinada persona en el seno de otra comunidad política. Este ejercicio elevaría esta idea más amplia de origen al quinto 
puesto del ranking, con 32 menciones (17 de la UE y 15 extracomunitarias). En cualquiera de los dos casos citados, como puede comprobarse, ni el puesto del motivo en la clasificación ni el número de menciones constitucionales supone un cambio especialmente notorio.

Tabla 5: Agrupaciones superiores de los ámbitos de discriminación previstos expresamente por al menos 20 constituciones europeas

\begin{tabular}{|l|l|l|l|l|l|l|}
\hline 1. Religión & EU & 21 & No EU & 18 & Total & 39 \\
\hline 2. Sexo & & 19 & & 17 & & 36 \\
\hline 3. Fenotipo & & 19 & & 16 & & 35 \\
\hline 4. Opinión/Convicción & & 18 & & 17 & & 35 \\
\hline 5. Origen & 17 & & 15 & & 32 \\
\hline 6. Lengua & 15 & & 12 & & 27 \\
\hline 7. Condición social & & 14 & & 12 & & 26 \\
\hline 8. Condición económica & & 9 & & 14 & & 23 \\
\hline 9. Otro & & 11 & & 9 & & 20 \\
\hline
\end{tabular}

En definitiva, y excluyendo la categoría residual de "otros motivos", los ejes de discriminación que están presentes en un número importante de constituciones (superior a 20) son los relativos a la religión, el sexo (género), el color de piel (raza), las opiniones y convicciones, el origen nacional o étnico, la lengua, y las condiciones sociales o económicas. Puede considerarse que en estos grupos de conceptos de incluyen las tres cuartas partes de las alusiones a motivos de discriminación de las constituciones europeas de hoy en día.

Un último análisis de la distribución de estos motivos en las constituciones europeas es el de la correlación entre constituciones de países próximos geográfica o culturalmente. Es decir, cabe preguntarse si se adivinan patrones consistentes entre diferentes constituciones europeas a la hora de listar los motivos de discriminación. El análisis de los mismos y su reiteración muestra que apenas existen patrones comunes en al ámbito de Europa occidental, seguramente por la mayor tradición constitucional de estos países, que provoca diferencias importantes en la edad de los respectivos textos fundamentales. Por ejemplo, los países nórdicos (Dinamarca, Finlandia, Islandia, Noruega y Suecia) no muestran ningún patrón común ni en la existencia de listados ni en el contenido de estos. Tampoco aparece similitud alguna al respecto en las constituciones de los países bálticos (Estonia, Letonia y Lituania). Descartamos también patrones comunes entre los tres Estados del sur del Cáucaso (Armenia, Azerbaiyán y Georgia), puesto que a pesar de que los tres muestran listados extensos (todos entre 14 y 17 motivos), solo coinciden realmente en 5 de los elementos citados, lo 
que muestra una frecuencia muy baja. Finalmente, tampoco parece haber coincidencias significativas entre los países de mayoría eslava o latina exsoviéticos (Bielorrusia, Moldavia, Rusia y Ucrania). Aparte del hecho de que Bielorrusia no incluye listado de motivos, los otros tres países solo coinciden en 4 de ellos, si bien resulta significativo que uno de ellos sea el relativo a la "propiedad".

Por el contrario, sí encontramos patrones similares entre Chequia y Eslovaquia, dos Estados separados de un mismo Estado precedente. A pesar de su diferente estructura constitucional, ambos países comparten en sus listados 12 de los 15 motivos que incluyen, lo que parece apuntar a una inspiración común. Por lo que respecta a los Estados surgidos de la antigua Yugoslavia (Bosnia-Herzegovina, Croacia, Eslovenia, Kosovo, Macedonia del Norte, Montenegro y Serbia), todos incluyen listados de entre 9 y 17 motivos, siendo los más frecuentes de 13 y 14 motivos. Entre éstos, todos los países citan, aparte de otros motivos más generalizados, el origen nacional o la nacionalidad, un factor muy relevante en la cultura política de la antigua Yugoslavia. Además, en este grupo aparecen numerosos motivos ligados al ámbito social y económico, todos los Estados menos uno (Eslovenia) citan el origen social; todos menos uno (Macedonia) el idioma; todos menos dos (Macedonia y Montenegro) el nacimiento; y muchos de ellos la propiedad y el estatus social. Estas similitudes también nos llevan a defender que existe una tradición común en este grupo de países a la hora de listar los motivos de discriminación constitucionalmente previstos. Los dos casos de la antigua Yugoslavia y la antigua Checoslovaquia parecen mostrar que los países desgajados de un único Estado mantienen ciertas pautas comunes a la hora de redactar sus constituciones, al menos en lo que a cláusulas antidiscriminatorias se refiere.

\subsection{Valoración y nuevos retos}

Los patrones coincidentes en el modo en que las constituciones europeas afrontan la prohibición de discriminación y los motivos de discriminación mencionados son el resultado de una evolución histórica. Los derechos fundamentales se fueron incorporando con lentitud a los textos constitucionales, estando ausentes de varias constituciones del siglo XIX ${ }^{11}$. En cuanto al derecho a la igualdad, la formulación elegida por cada ordenamiento ha dependido tanto del texto constitucional que lo incorporaba como de la historia política y social del país ${ }^{12}$. Desde un punto de vista exclusivamente normativo, la determinación de los elementos protegidos o motivos de discriminación potencial opera como resultado de una tensión creativa entre diferentes fuentes, entre las que se encuentran las normas constitucionales, la interpretación judicial o las normas internacionales de derechos humanos ${ }^{13}$. En este sentido, se produce una constante interrelación entre estos tipos de fuentes que deriva en migra-

\footnotetext{
11 PEGORANO, L. y RINELLA, A., cit., p. 169.

12 O'REGAN, K. y FRIEDMAN, N. (2011), cit., p. 473.

13 FREDMAN, S., cit., p. 111.
} 
ciones dentro del propio Derecho constitucional comparado. Sujit Choudhry usa la migración como metáfora, recordando que existe cada vez mayor jurisprudencia comparada y mayor dialogo entre tribunales de distintas instancias, de forma que el Derecho constitucional comparado debe también analizar la migración de las ideas constitucionales de unos países a otros ${ }^{14}$.

Las constituciones europeas actualmente vigentes tienen su origen en varios momentos históricos. Desde los inicios decimonónicos de las constituciones de Noruega, Dinamarca o los países del Benelux hasta las numerosas que tienen un origen reciente. De hecho, son mayoría las constituciones analizadas que no alcanzan las tres décadas de vigencia. Son escasas las que tienen su origen en la época de entreguerras (Austria, Irlanda, Letonia o Liechtenstein) y un grupo mayor de constituciones occidentales provienen de las décadas posteriores a la segunda guerra mundial, mientras que en la Europa central y oriental abundan las constituciones de última generación. A ello hay que añadir las numerosas reformas que han sufrido las constituciones europeas más antiguas.

Con independencia de su origen, las constituciones se encuentran sometidas al cambio y la evolución, e íntimamente relacionadas con las políticas que pueden ayudar a consolidarlas o a transformarlas ${ }^{15}$, de forma que van incorporando ideas y demandas de su contexto social y político sin una base normativa previa, generando en ocasiones nuevas migraciones constitucionales. Así, por ejemplo, entre los motivos de discriminación que hemos detectado como más frecuentes en el estado actual del Derecho constitucional comparado hay algunos que tienen un largo recorrido histórico, mientras que otros se han incorporado de manera mucho más reciente, sea por influencia de jurisdicciones ajenas o por la mera presión social interna. En el presente existe gran consenso sobre el hecho de que el sexo y los rasgos fenotípicos (raza) deben estar en dichos listados (no en vano son el segundo y tercer motivo más citados en Europa conforme a nuestro análisis previo), pero ambos fueron incorporados a las constituciones después de un largo combate y de manera relativamente tardía. En el caso de la raza, solo después de la segunda guerra mundial, mientras que las discriminaciones basadas en las diferencias entre sexos o géneros han sido mayoritariamente incorporadas a los listados en los últimos 20 años, junto con numerosas cláusulas explícitas de igualdad entre hombres y mujeres. Esto no obsta a que se haya convertido en poco tiempo en el segundo motivo agregado más citado por el conjunto de constituciones europeas. También es relativamente reciente la incorporación de motivos relacionados con la salud, la predisposición genética o las diversidades funcionales

${ }^{14}$ CHOUDHRY, S. (ed.)(2006), The Migration of Constitutional Ideas, Cambridge, Cambridge University Press, p. 16.

15 HERINGA, A.W. (2016), Constitutions Compared. An Introduction to Comparative Constitutional Law, 4th ed., Cambridge, Intersentia, p. 261.

(C) UNED. Revista de Derecho Político

N. ${ }^{\circ} 107$, enero-abril 2020, págs 41-69 
de las personas ${ }^{16}$ o con la orientación sexual, aún poco presente de manera explícita ${ }^{17}$.

Podemos constatar que no existe relación directa entre la antigüedad de un motivo de discriminación y su frecuencia de aparición en las constituciones europeas. En este sentido, las constituciones de nueva generación no aportan un elemento sustancialmente diferente a los listados de motivos de discriminación. Su contribución consiste normalmente en aumentar el número de motivos expresamente citados, pero las nuevas menciones vienen a coincidir con las que las reformas de las constituciones más antiguas van incorporando. En el diálogo interconstitucional de hoy en día no puede concluirse que las segundas son reformadas por influencia de las primeras, aunque sí puede advertirse que las nuevas constituciones tienden a incorporar un mayor número de motivos y a agotar los listados por la mayoría de las restantes constituciones europeas. En esta dinámica, el número total de motivos de discriminación en las constituciones es creciente.

Aunque el objeto de este análisis es la comparación constitucional, es preciso aludir a la existencia de varias normas internacionales que prohíben la discriminación y que también incorporan cláusulas similares a las aquí analizadas. No en vano el plano normativo internacional constituye, como se señalaba, una de las fuentes de inspiración constitucional en este ámbito. Las disposiciones relevantes se contienen en los tratados de protección de derechos humanos de las Naciones Unidas ${ }^{18}$, pero también en tratados del Consejo de Europa ${ }^{19}$ y del ámbito de la Unión Europea para sus países miembros ${ }^{20}$. Sin duda estos tratados internacionales han ejercido una influencia sobre los motivos de discriminación que se han adoptado constitucionalmente. De hecho, un análisis comparativo similar a éste sobre la base de estos tratados arro-

${ }^{16}$ La discriminación genética (predisposición a padecer una enfermedad genética) fue reconocida en Estados Unidos en 2008: SOLANKE, I. (2017), Discrimination as Stigma. A Theory on Anti-discrimination Law, Oxford-Portland, Hart Publishing, p. 44.

17 FREDMAN, S., cit., p. 109.

18 Principalmente, la Convención Internacional sobre la Eliminación de todas las Formas de Discriminación Racial de 1965 (artículo 1), el Pacto Internacional de Derechos Civiles y Políticos de 1966 (artículos 2 y 26), el Pacto Internacional de Derechos Económicos, Sociales y Culturales de 1966 (artículo 2), la Convención sobre la eliminación de todas las formas de discriminación contra la mujer de 1979 (artículo 1), la Convención contra la Tortura y Otros Tratos o Penas Crueles, Inhumanos o Degradantes de 1984, la Convención sobre los Derechos del Niño de 1989 (artículo 2), la Convención sobre los derechos de las personas con discapacidad de 2006 (artículo 5) y la Convención internacional sobre la protección de los derechos de todos los trabajadores migratorios y de sus familiares de 1990 (artículos 1 y 7). Desgraciadamente, esta última sigue teniendo un número de ratificaciones muy bajo entre los países europeos.

19 Principalmente, el Convenio Europeo de Derechos Humanos (1950)(artículo 14 y Protocolo número 12).

${ }^{20}$ Tratado de Funcionamiento de la Unión Europea (artículo 10, incorporado en 2009) y la Carta de los Derechos Fundamentales de 2000(artículo 21), 
jaría resultados muy similares respecto a la frecuencia de los motivos de discriminación citados expresamente ${ }^{21}$.

En la valoración de los datos comparados resulta más relevante la estructura de las cláusulas antidiscriminatorias. Como ya sabemos, no todas las constituciones europeas incluyen listados de motivos de discriminación (39 de 50) y la mayor parte de las que lo hacen (20) incorporan un motivo residual que deja la puerta abierta con carácter permanente al ajuste interpretativo por vía doctrinal o jurisprudencial. Otro elemento de importancia para este análisis es que las constituciones no incluyen referencias expresas a las discriminaciones múltiples que puedan producirse, sea en modo acumulativo o interseccional, a pesar de la relevancia que estos conceptos han adquirido al demostrar la existencia de discriminaciones no cubiertas por los cánones clásicos de la aplicación individualizada de motivos. Sería conveniente que las constituciones empezaran a incorporar referencias a diversas suertes de discriminación en las que concurren varios motivos, toda vez que ello supone una mejor protección de determinados colectivos (o subgrupos) que han quedado tradicionalmente invisibilizados en la dinámica clásica del test de comparación (por ejemplo, el arquetípico caso

${ }^{21}$ Hemos analizado y comparado, siguiendo el mismo método usado con las constituciones, los listados presentes en los siguientes nueve textos: Declaración Universal de Derechos Humanos (art. 2), Pacto Internacional de Derechos Civiles y Políticos (art. 26, que repite los motivos listados en su artículo 2), Convención Internacional sobre la Eliminación de todas las Formas de Discriminación Racial (artículo 1), Pacto Internacional de Derechos Económicos, Sociales y Culturales (art. 2), Convención sobre los Derechos del Niño (art. 2), Convención internacional sobre la protección de los derechos de todos los trabajadores migratorios y de sus familiares (art. 7, que repite el listado de su artículo 1), Tratado de Funcionamiento de la Unión Europea (art. 10), Carta de los Derechos Fundamentales de la Unión Europea (art. 21), y Convenio Europeo para la Protección de los Derechos Humanos y de las Libertades Fundamentales (art. 14). Otros cuatro textos del ámbito institucional de las Naciones Unidas no ofrecen un listado de motivos de discriminación por razón de su especificidad temática: Convención sobre la eliminación de todas las formas de discriminación contra la mujer, Convención contra la Tortura y Otros Tratos o Penas Crueles, Inhumanos o Degradantes, Convención Internacional para la protección de todas las personas contra las desapariciones forzadas y Convención sobre los derechos de las personas con discapacidad.

El listado completo de motivos que aparecen citados en uno o varios de estos nueve tratados es el siguiente: raza, color, sexo, idioma/lengua, religión (o convicción), opinión política o de cualquier otra índole (u otras), origen nacional, nacionalidad, origen étnico, pertenencia a una minoría nacional, origen social, nacimiento, linaje, posición económica/situación económica, patrimonio, fortuna, estado civil, edad, características genéticas, impedimentos físicos/discapacidad, orientación sexual, cualquier otra condición (social)/cualquier otra situación.

Su frecuencia de aparición coincide con los resultados obtenidos para las constituciones y reflejados en la tabla 5 de este mismo trabajo, de forma que aparecen con el número máximo de citas (9) el fenotipo y el origen; con 8 menciones la religión, el sexo y la condición social; y con 7 apariciones la opinión/ convicción, la lengua y la condición económica. Estos ejes coinciden con los ocho primeros que presentaba la mencionada tabla 7 , dejando el noveno puesto para la cláusula residual (6). Sensiblemente por debajo de esta cifra, solo aparecen citados la discapacidad/impedimentos físicos (3), la edad (3), la orientación sexual (2), las características genéticas (1) y el estado civil (1). 
de las mujeres afroamericanas, ya denunciado por algunas autoras desde los años ochenta y noventa ${ }^{22}$ ).

En este marco, puede resultar muy relevante no solo la existencia de un listado expreso que mencione motivos de discriminación expresamente excluidos, sino también la incorporación de una cláusula residual que permite incluir en la norma otras situaciones no expresamente citadas. Esta diferencia adquiere su importancia precisamente ante el fenómeno de las discriminaciones interseccionales. En nuestra opinión, la presencia de una cláusula abierta en el listado permite incluir discriminaciones interseccionales que de otro modo pueden quedar fuera del análisis judicial, por lo que valoraríamos las cláusulas con listados abiertos de una manera más positiva que las cláusulas con listados cerrados o sin listado. Esta mayor operatividad de las cláusulas abiertas se puede observar en la diferente disposición que el Tribunal Europeo de Derechos Humanos y el Tribunal de Justicia de la Unión Europea han demostrado a la hora de acoger la idea de discriminación múltiple. Así, el hecho de que el artículo 14 del Convenio Europeo de Derechos Humanos incluya un motivo residual ha posibilitado que el Tribunal de Estrasburgo haya usado la categoría de discriminación acumulativa o interseccional. Por el contrario, el Tribunal de Luxemburgo se ha mostrado hasta el momento reacio a ello, no encontrando en las directivas de igualdad actualmente vigentes una cláusula abierta ${ }^{23}$. Desde esta lógica, es clara la opción por un modelo de cláusula que incluya una lista de motivos abierta que permita incluir más fácilmente situaciones discriminatorias actuales que no fueron contempladas cuando la norma constitucional fue escrita.

Más allá del modelo de cláusula más efectivo, debemos también preguntarnos por los nuevos motivos de discriminación que deban surgir o consolidarse en el futuro. Sabemos que aunque hoy en día el dialogo interconstitucional es más fértil que en épocas anteriores, las sociedades se transforman con creciente velocidad. Se trata de plantear hasta qué punto estos listados actuales de motivos de discriminación resultan útiles de cara a transformaciones sociales futuras, o cuál debe ser la dinámica de incorporación de nuevos motivos para garantizar, como pretenden las constituciones democráticas, la protección de la dignidad humana. Tal y como se pregunta Fredman, ¿existe una dinámica unificadora que explica y nos permite predecir la incorporación de motivos de discriminación o este proceso es simplemente el resultado de la confluencia de factores sociales, históricos y políticos? ${ }^{24}$.

Ante esta cuestión no puede aventurarse una respuesta fácil ni unívoca en el estado actual del Derecho constitucional comparado. Ni en el patrón de motivos de discriminación explícitamente incorporados ni en la evolución temporal de los mismos podemos identificar un principio uniformizador, lo que nos llevaría a otorgar

22 WILLIAMS CRENSHAW, (1991), "Mapping the Margins: Intersectionality, Identity Politics, and Violence against Women of Color", Stanford Law Review, 43-6, pp. 1245-1250

${ }^{23}$ LOUSADA AROCHENA, J.F. (2017), "Discriminación múltiple: El estado de la cuestión y algunas reflexiones", AequAlitas, 41, pp. 35-36.

${ }^{24}$ FREDMAN, S., cit., p. 113. 
una respuesta negativa a la pregunta y a insistir en una permanente contextualización de los textos constitucionales. Hay quien sostiene que quizás en vez de abandonar la búsqueda de un principio unificador sobre quién merece protección o sobre qué motivos deba sustentarse dicha protección constitucional, la búsqueda puede ser reformulada, transitando instrumentalmente desde la dignidad humana al estigma social y el estereotipo ${ }^{25}$. O, lo que es lo mismo, desde plantear la cuestión como una pregunta filosófica o moral a realizarla desde el campo de la psicología social. El estigma o el estereotipo podrían así proveernos con la base para comprender qué características necesitan protección. Ello reforzaría una respuesta basada en la dinámica social o política de cada contexto. Y nos obligaría a comprender cómo se detecta el estigma social y cómo se previene a través de normas jurídicas (u otro tipo de políticas). Seguramente no nos resultaría fácil adelantarnos a los procesos de estigmatización, o a la identificación de los mismos, y las normas constitucionales posiblemente seguirían constituyendo soluciones reactivas a fenómenos que ya se estaban produciendo y que en un momento dado son percibidos como reprobables social o políticamente.

Partiendo de la opción por cláusulas amplias, con listados detallados y abiertos que permitan una interpretación de la discriminación lo más comprensiva posible, es necesario plantearse si aquéllas seguirán constituyendo un arma jurídica eficaz ante posibles prácticas discriminatorias derivadas de las potencialidades de nuevas tecnologías de comunicación e información. Los cambios derivados de algunos avances tecnológicos en ámbitos como la ingeniería genética, la inteligencia artificial o el tratamiento de datos pueden resultar muy relevantes para el Derecho antidiscriminatorio. Posiblemente el elemento más perturbador es que las discriminaciones podrán basarse no tanto en la existencia de uno o varios elementos personales característicos de unos determinados colectivos sociales (lo que aquí hemos llamado motivos de discriminación), sino en un cruce inmenso de datos diferentes que a través de un algoritmo van a determinar una condición individual. Las discriminaciones que puedan producirse en el acceso al empleo, al alquiler de una vivienda o a la contratación de un seguro médico podrán basarse ya no en motivos de discriminación como los que recogen nuestras constituciones, y que podríamos considerar colectivos, sino en condiciones individuales que derivan de una pléyade de datos entre los que se encontrarán algunos de los actuales listados, pero con un grado mucho mayor de precisión. Como señala lúcidamente Harari, en este nuevo mundo saturado de datos personales procesados "el algoritmo nos discrimina no porque seamos una mujer o un afroamericano, sino porque somos nosotros. Hay algo específico en nosotros que no le gusta. No sabemos qué es, y aunque lo supiéramos no podríamos organizarnos con otras personas que padezcan el mismo prejuicio exacto. Solo nosotros. En vez de simplemente dis-

25 SOLANKE, I. (2017), cit., p. 62.

(C) UNED. Revista de Derecho Politico 
criminación colectiva, en el siglo XXI podríamos enfrentarnos a un problema creciente de discriminación individual" 26 .

Lo que el historiador israelí llama aquí “discriminación individual” no responde a una definición jurídica y en principio faltaría en ella el elemento comparativo colectivo que sustenta la discriminación. Al contrario, esta suerte de práctica potencialmente discriminatoria se produce tras una ponderación muy detallada de diversos elementos de adscripción a hipotéticos grupos de pertenencia no perfectamente definidos, pero que parecen asociarse a cuestiones ligadas a la edad, apariencia, condición física o mental, salud, carácter o comportamiento emocional. Es cierto que algunas de estas características aparecen en los listados constitucionales, pero lo hacen siempre en consideración a un momento determinado (por ejemplo, no se considera "persona con discapacidad" a aquélla que aún no ha desarrollado determinada patología limitativa, y sin embargo hoy en día es posible saber que la desarrollará en el futuro y por tanto rechazar su candidatura en atención a dicho dato). Además, la combinación y ponderación de elementos que permiten las tecnologías es tan amplia y específica que nos resulta imposible asociar el trato discriminatorio a un único factor. En este sentido, este tipo de prácticas se acercarían a una suerte de discriminación múltiple que pondera numerosos factores de manera distinta para cada persona. La dinámica apunta en realidad no ya a la determinación de subgrupos discriminados que emergen al aplicar el filtro de la discriminación interseccional, sino a la creación de infinitos subgrupos, tantos como personas, potencialmente discriminados (una suerte de "interseccionalidad extrema o infinita”). ¿Será posible combatir jurídicamente estas prácticas acudiendo al concepto actual de discriminación múltiple y valiéndonos para ello de las cláusulas abiertas? Es sin duda la vía más eficaz de las actualmente existentes. Del mismo modo que sucede con la interseccionalidad, será necesario avanzar desde una apreciación de la discriminación estrictamente basada en un test de comparación hacia un análisis fundado sobre un test de perjuicios, paliando la inexistencia de un colectivo perfectamente predefinido por la confluencia de varios motivos de discriminación ${ }^{27}$.

Es preciso combatir estas posibles prácticas discriminatorias desde las normas actuales, pero al mismo tiempo es necesario repensar las cláusulas en su formato actual o valorar la posible incorporación de motivos nuevos que prevean estas utilizaciones no deseadas del aluvión de datos personalizados que pueden hoy utilizarse para una multitud de fines. Estas y otras amenazas a la igualdad no están aún presentes de manera explícita en nuestros desarrollos normativos y deberán ocupar la atención doctrinal y política de cara a la siguiente generación de motivos de discriminación y a la estructura de las cláusulas constitucionales antidiscriminatorias.

${ }^{26}$ HARARI, Y.N. (2018), 21 lecciones para el siglo XXI, Barcelona, Penguin Random House Grupo Editorial, Barcelona. P. 91.

27 LOUSADA AROCHENA, J.F. (2017), cit., pp. 29-40. 


\section{CONCLUSIONES}

En la actualidad, 42 de las 50 constituciones europeas incluyen una cláusula antidiscriminatoria expresa. La estructura típica de dichas cláusulas consiste en la afirmación del derecho a la igualdad seguida de la prohibición de la discriminación sobre la base de una serie de motivos concretos. Hasta 39 constituciones nos ofrecen listados de los motivos de discriminación o elementos protegidos específicamente. De estos listados, algo más de la mitad se plantean un motivo residual que pueda incorporar otras circunstancias no señaladas expresamente. Estas últimas cláusulas resultan las más eficaces en la actualidad puesto que dejan mayor margen para incorporar nuevas formas de discriminación, en particular discriminaciones múltiples, tanto cumulativas como, sobre todo, interseccionales, que se producen precisamente por la combinación de varios motivos de discriminación y no en su mero análisis por separado.

A su vez, muchas constituciones europeas incluyen referencias expresas a la igualdad entre hombres y mujeres que van más allá de incluir el género o sexo como un motivo de discriminación. La mayor parte de estas referencias han sido incorporadas en reformas constitucionales recientes o por constituciones relativamente jóvenes. En cualquier caso, la inclusión de una referencia expresa a la igualdad entre géneros parece confirmarse ya como parte del acervo constitucional común de los países europeos.

Por lo que respecta a los elementos que centran la prohibición de discriminación en las constituciones europeas, puede señalarse que, en efecto, existen tendencias bastante consistentes en el continente. Los motivos de discriminación que están ampliamente presentes en las constituciones europeas (y en las cláusulas correspondientes de los tratados internacionales) son los relativos a la religión, el sexo o género, el color o raza, las opiniones y convicciones, el origen nacional o étnico, la lengua, y las condiciones sociales o económicas. Esto constata, por una parte, que existen tendencias comunes (migraciones) al respecto en el constitucionalismo actual, europeo en particular. Por otra parte, que hoy en día se entiende que los mayores riesgos de discriminación, o los elementos de pertenencia colectiva que es necesario proteger especialmente son los incluidos en este listado.

Ahora bien, el objetivo del Derecho constitucional comparado no consiste únicamente en organizar sistemáticamente el conocimiento en su ámbito, investigando y analizando las analogías y diferencias entre textos u ordenamientos constitucionales. Más allá de mostrar una finalidad meramente especulativa, el Derecho constitucional comparado está llamado a influir sobre instituciones, procedimientos y sobre los propios textos normativos ${ }^{28}$. En este sentido, la configuración de las cláusulas constitucionales antidiscriminatorias responde a un modelo que podemos considerar clásico, más allá de que los motivos de discriminación puedan irse actualizando mediante

${ }^{28}$ PEGORANO, L. y RINELLA, A., cit., pp. 1117-118.

(C) UNED. Revista de Derecho Politico

N. ${ }^{\circ}$ 107, enero-abril 2020, págs 41-69 
procesos de reforma o mutación. Este modelo no ha incorporado definitivamente la discriminación múltiple, sobre todo en su vertiente interseccional y puede plantear dudas de eficacia ante posibles discriminaciones de nuevo cuño sustentadas no en datos genéricos de concurrencia de los motivos ya citados por las constituciones, sino en su combinación o ponderación con gran precisión sobre el tratamiento de numerosos datos personales (biométricos, emocionales...) que hoy en día pueden generar prácticas de mayor dificultad probatoria. El Derecho antidiscriminatorio en general, y las constituciones en particular, deberán explorar los mecanismos que puedan prevenir y combatir eficazmente las nuevas formas de discriminación no basadas en elementos colectivos reconocibles socialmente. Será necesario para ello avanzar en las cláusulas abiertas y explorar la forma de otorgar mayor concreción a la discriminación por combinación múltiple y específica de motivos presentes o futuros.

\section{REFERENCIAS}

Chopin, I. y Germaine, C. (2017), A comparative analysis of non-discrimination law in Europe. The 28 EU Member States, the Former Yugoslav Republic of Macedonia, Iceland, Liechtenstein, Montenegro, Norway, Serbia and Turkey compared (prepared for the European network of legal experts in gender equality and non-discrimination), Brussels, European Commission (Directorate-General for Justice and Consumers).

Choudhry, S. (ed.)(2006), The Migration of Constitutional Ideas, Cambridge, Cambridge University Press.

Fredman, S. (2011), Discrimination Law, $2^{\text {nd }}$ ed., Oxford, Oxford University Press.

Ginsburg, T. y Dixon, R. (eds.)(2011), Comparative Constitutional Law, Cheltenham, Edward Elgar.

Harari, Y.N. (2018), 21 lecciones para el siglo XXI, Barcelona, Penguin Random House Grupo Editorial, Barcelona.

Heringa, A.W. (2016), Constitutions Compared. An Introduction to Comparative Constitutional Law, 4th ed., Cambridge, Intersentia.

Lousada Arochena, J.F. (2017), “Discriminación múltiple: El estado de la cuestión y algunas reflexiones”, AequAlitas, 41, 29-40.

Mccolgan, A. (2000), Discrimination Law. Text, cases and materials, Oxford-Portland Hart Publishing.

Mccolgan, A. (2014), Discrimination, Equality and the Law, Hart Publishing 2014

O'regan, K. y Friedman, N. (2011), "Equality", en Ginsburg, T. y Dixon, R. (eds.), Comparative Constitutional Law, Cheltenham, Edward Elgar, 473-503.

Osin, N. y Porat, D. (eds.)(2005), Legislating against Discrimination. An international Survey of Anti-Discrimination Norms, Leiden-Boston, Martinus Nijhoff Publishers.

Pegorano, L. y Rinella, A. (2007), Diritto Pubblico Comparato. Profili metodologici, Padova, CEDAM.

66

(C) UNED. Revista de Derecho Politico

N. ${ }^{\circ} 107$, enero-abril 2020, págs 41-69 
Solanke, I. (2017), Discrimination as Stigma. A Theory on Anti-discrimination Law, Oxford-Portland, Hart Publishing.

Williams Crenshaw, K. (1991), "Mapping the Margins: Intersectionality, Identity Politics, and Violence against Women of Color", Stanford Law Review, 43-6, 1241-1299.

\section{Title}

Antidiscrimination clauses and prohibited grounds of discrimination in European Constitutions.

\section{Summary}

1.- Introduction. 2.- The comparison of antidiscrimination clauses in Constitutions: methodological difficulties. 3.- Antidiscrimination clauses in European Comparative Constitutional Law. 3.1.- Models and structures. 3.2.- Existing prohibited grounds of discrimination. 3.3.- Evaluation and new challenges. 4.- Conclusions 5.- References.

\section{Resumen}

Casi todas las constituciones europeas incluyen referencias expresas a la prohibición de discriminación, normalmente a través de la incorporación de cláusulas específica y exclusivamente orientadas a ello. A su vez, en la mayoría de dichas disposiciones constitucionales se incorporan listados de los factores o elementos que se consideran especialmente proclives a motivar una discriminación o diferencias que meritan una especial protección por parte del ordenamiento jurídico.

Comparar y sistematizar los motivos concretos de discriminación que las constituciones europeas mencionan plantea en primer lugar una serie de dificultades metodológicas sobre las que es preciso advertir. Además de las barreras lingüísticas propias del análisis de textos producidos en idiomas diferentes, es necesario definir el ámbito de estudio tanto por la vertiente normativa como por la geopolítica. Una vez realizadas las necesarias acotaciones conceptuales previas, puede concluirse la existencia de ciertos patrones comunes tanto en cuanto a la redacción de las cláusulas constitucionales antidiscriminatorias como en cuanto a los listados de las diferencias expresamente protegidas o motivos de discriminación.

Así, en la actualidad, muchas constituciones europeas incluyen referencias expresas a la igualdad entre hombres y mujeres, más allá de la inclusión de factores como el género o sexo entre los motivos explícitos de discrimina-

(C) UNED. Revista de Derecho Político 
ción. Por lo que respecta a éstos, los factores cuya consideración se considera discriminatoria más ampliamente citados en las constituciones europeas incluyen la religión, el sexo o género, los rasgos fenotípicos, la lengua, las opiniones y convicciones, las condiciones sociales o económicas y el origen nacional o étnico. La posible agrupación de al menos tres cuartas partes de los motivos citados en torno a los factores mencionados demuestra patrones muy comunes al respecto en el Derecho constitucional comparado. Al mismo tiempo, solo parte de las constituciones incluyen cláusulas abiertas o motivos residuales, lo que puede dificultar la apreciación de discriminaciones múltiples de tipo interseccional. Es preciso también adaptar estas normas y su interpretación ante posibles discriminaciones futuras basadas en el uso masivo de datos individuales referidos simultáneamente a varios motivos de discriminación.

\begin{abstract}
Almost all European constitutions include explicit references to the prohibition of discrimination, usually through the inclusion of exclusive and specific clauses In addition, most of these constitutional dispositions include a list of the factors or elements that can lead to discrimination.

The current paper offers a comparison and systematization of the protected grounds of discrimination mentioned by the European constitutions, and which entail a number of methodological difficulties. Beyond the linguistic obstacles that analysing texts in very different languages present, it is necessary to define the scope of analysis, both from a legal and geopolitical perspective. Once the conceptual limits are defined, we can observe common patterns in respect to both the wording and structure of the antidiscrimination constitutional clauses as well as to the lists of prohibited grounds of discrimination.

Nowadays most European constitutions make explicit reference to the principle of equality between men and women, as well as to the inclusion of the factor of sex or gender as one of the explicitly mentioned prohibited grounds. As for these prohibited grounds, the most widely mentioned antidiscrimination prohibited grounds of discrimination in European constitutions are religion, sex or gender, phenotypical features, language, opinions and convictions, social or economic conditions and national or ethnic origin. The fact that over $75 \%$ of the explicit grounds fall within the aforementioned groupings provide support for the need to analyse existing common patterns in European constitutions. At the same time, only some of the constitutions include open clauses or grounds and this factor may hinder the determination of multiple or intersectional discriminations. It is also necessary to adapt these clauses and their interpretation to face possible future discriminations based on the massive use of personal data referring simultaneously to several discrimination grounds.
\end{abstract}




\section{Palabras clave}

Discriminación / Constituciones / Derecho comparado / Motivos de discriminación / Cláusulas antidiscriminatorias

\section{Keywords}

Discrimination / Constitutions / Comparative Law / Prohibited Grounds of Discrimination / Antidiscrimination Clauses 